\title{
Remedy Selection based on Artificial Intelligent Methods
}

\author{
Saeedeh Sadat Sadidpour \\ M.Sc. in Computer Engineering and B.Sc. in \\ Homeopathy \\ AmirKabir University of Technology \\ Iran
}

\author{
Saeed Shiry Ghidary \\ Assistant Professor \\ Computer Engineering Department \\ AmirKabir University of Technology \\ Iran
}

\begin{abstract}
Homeopathy decision is a decision under uncertainty and an essential activity for accurate treatment. Expert systems have long history of application in medical diagnosis. In this paper we study application of Fuzzy Expert System and decision tree for selection of suitable remedy in Homeopathy. A decision made by Homeopath is highly dependent on the quality of selected rubrics. The rubrics are collected during case taking and generally contains expressions that might be considered fuzzy, such as 'never', 'sometimes', 'always', and so on, making it difficult to model them with conventional computational methods. In this context, fuzzy set theory in expert systems is an interesting tool to deal with the representation of inaccurate medical entities. Hence, we can go from natural language (linguistic variables) to numerical variables which are more convenient to handle in a computer. The proposed method reduces sensitivity of system to homeopath mistakes and increase security of system.
\end{abstract}

\section{General Terms}

Homeopathy, Intelligent Systems, Remedy Selection.

\section{Keywords}

Intelligent diagnosis, Decision Tree, Fuzzy Expert System, Homeopathy, Remedy Determination.

\section{INTRODUCTION}

Materia Medica is the primary source of information in homeopathy. The information about homeopathic remedies is explained by Materia Medica. Homeopathic proving, research, and clinical information are some ways to reach the information of remedies. Regardless of the huge discovered information, the homeopaths often prescribe limited remedies. The basic drawbacks of the Materia Medica are:

- $\quad$ Large amounts of information cannot be completely and effectively perceived.

- The use of one Materia Medica provides only incomplete information.

- The use of many Materia Medica makes available duplicate information.

- The search through Materia Medica is very timeconsuming.

- The authors of Materia Medica use different languages and words. As a result, a mean is explained differently.

- The amount of available information in Materia Medica is huge. Therefore, a more effective structuring is necessary.

The homeopathic repertory classifies the information of remedies. The information was restructured by the repertory to be used more effectively and help homeopath in the process of remedy prescription. The homeopath is able to choose the symptoms of the patient and (s)he should have the ability of distinguishing all homeopathic remedies which are related to the issue.

Although the reversed informational structure of the homeopathic repertory solves some of the conversing problems in the extent of Materia Medica, it has some new problems. The repertory structure also has its special rules for retaining the consistency of the contained data. The search of repertory is time-consuming and a computer aided search algorithm helps homeopath very much [1].

Artificial intelligence is a part of computer science that tries to make computers more intelligent. The learning is a basic requirement for any intelligent behavior. Therefore, machine learning [2] is a major branch, and the most rapidly developing subfield of artificial intelligence research.

Machine learning algorithms have been used to analyze medical data sets. Some essential tools are provided to analyze intelligent data by these algorithms. The digital systems also collect and store the data using relatively inexpensive and available means. Modern hospitals are equipped with monitoring and other data collection devices. The large information systems gather and share the data. Machine learning technology is used to analyze medical data. As a result, a lot of medical diagnosis is done in small specialized diagnostic problems [3].

The description of solved cases is leaded to medical diagnostic knowledge. The achieved classifier are used to assist the physician to diagnose with more speed, accuracy and reliability, and to train the students or physicians nonspecialists [4].

Fuzzy logic is an artificial intelligence method which is similar to human thinking. A specific parameter is shown by several states. Each state changes in boundaries and a case is a member of a parameter with a probability.

Fuzzy expert system is an expert system that uses fuzzy content. The systems not only have some if-then rules, but also use from rules with membership degree.

In this paper, at first, we explain about source of information in homeopathy and usage of machine learning in medical diagnosis. At next section, a brief history of working with machine learning for medical diagnosis is described. Then, machine learning methods and how to use them for homeopathy is described. And finally, conclusion is presented.

\section{HISTORICAL OVERVIEW}

The medical diagnostic decision by computer systems is possible if the diagnostic process is done as logical analysis. The development of these computer systems is part of the research field called artificial intelligence, which is defined as "the branch of computer science that is concerned with the automation of intelligent behavior". The ability of computers 
is increased to process, store data, and carry out the complex analysis. These computers are able to make data and results in association with networks available quickly at a distance. Many classic expert systems have used the mathematical and statistical methods including decision trees, discriminate analysis and cluster analysis. More recently, the fuzzy sets theory is another method that is noteworthy and has been applied in medicine. The fuzzy sets especially fuzzy expert systems have been used successfully in medicine diagnosis.

The interpretation of the natural language is an essential work for the creation of decision support systems. This approach is ordinarily used in clinical practice. The patients often describe the signs and symptoms with common but subjective terms such as: "worse", "better", "stable", "a little", "a lot", "moderate", "discretely" and "intense". They even use the no less subjective cross code or exemplify, therefore, the vagueness of data is increased. The fuzzy sets theory is a prominent progress to deal with such extreme inexactness $[5$, $6]$.

Adlassnig described a new concept for a system based on fuzzy set theory in 1980. The system formalize symptoms and diseases using fuzzy logic [5]. The new system, CADIAG-II, was implemented in 1982, and completely incorporated into the medical information system WAMIS in 1984. CADIAG-II was made better over the following years [7].

In homeopathy field, Prof. George Vithoulkas created the V.E.S. (Vithoulkas Expert System) in co-operation with the University of Namur. V.E.S. is used to analysis the homeopathic cases. The V.E.S. follows the line of thought of Prof. Vithoulkas. The Radar software has profited V.E.S. in Archibel. The symptoms are considered with intensities of 1 to 4 . Intensities 1 and 2 are for symptom clearly present and symptom very frequent or very intense. Intensities 3 and 4 are also for symptom very frequent and very intense, and symptom very, very frequent and intense, respectively [8].

\section{DECISION TREE LEARNING}

The decision tree method is a common technique used in data mining. Each interior node corresponds to a variable. A possible value of a variable is shown by an arc to that child. The value of target variable is expressed by a leaf. The values of the variables are represented by the path from the root to the leaf.

The tree learning process is a recursive manner. The source set is split into subsets based on an attribute value test. Then a tree is learned by the repeated process on each derived subset. The recursion is done until splitting is impossible, or due to a singular classification. In order to improve the classification rate, a number of decision trees are used as a random forest classifier.

Trees are also described as the combination of mathematical and computing techniques in data mining. The techniques aid the description, categorization and generalization of a given set of data. Data comes in records of the form:

$$
(x, y)=\left(x_{1}, x_{2}, \cdots, x_{k}, y\right)
$$

$y$ is the dependent variable we are trying to understand, classify or generalize. The other variables $x_{1}, x_{2}$, etc., are the variables that will help with that task [9].

\section{FUZZY SETS THEORY}

Prof. Lotfi A. Zadeh, from University of California, Berkeley, introduced the theory of fuzzy sets in the 1960's [10]. The theory of fuzzy sets models the uncertainty within natural language. The mechanism of fuzzy sets theory was advertised in 1965, based on key notion of graded membership which Zadeh had represented. In this approach, members of a set belong to it only in part. Such fuzzy sets have imprecise boundaries. As a result, an element transits gradually in its fuzzy set from membership to non-membership. The interaction of natural language and numerical models is provided by fuzzy sets.

In addition, if we assume that $X$ is a set serving as the universe of discourse, a fuzzy subset A of $\mathrm{X}$ is associated with a characteristic function:

$$
\mu_{\mathrm{A}}: x \rightarrow[0,1]
$$

which is generally called membership function. Then, for each $x$, value of $\mu_{\mathrm{A}}(x)$ indicates to what extent $\mathrm{x}$ is a member of set A. This membership degree indicates the compatibility degree of the assertion " $x$ is $A$ ". For example, if a body temperature is above $37.0^{\circ} \mathrm{C}$, (s)he has a fever. Thus, a patient with $38^{\circ} \mathrm{C}$ has a fever (membership degree of 1.0 ). But two patients with temperatures of $36.8^{\circ} \mathrm{C}$ and $37.0^{\circ} \mathrm{C}$ are members of the "febrile patient set" too with a membership degree of 0.8 and 0.9 , respectively, if we use the fuzzy sets theory.

The classic set theoretical operations are developed to fuzzy sets, which have membership degrees in the interval (0.1). So, if $\mathrm{A}$ and $\mathrm{B}$ are two fuzzy subsets of $\mathrm{X}$, their union is a fuzzy subset $\mathrm{C}$ of $\mathrm{X}$, denoted $C=A \cup B$ identified by the maximum (max) value between $A(x)$ and $B(x)$, such that for each $\mathrm{x}$ in $\mathrm{X}$ :

$$
\mathrm{C}(\mathrm{x})=\operatorname{Max}[A(x), B(x)]
$$

In addition, their intersection is another fuzzy subset $\mathrm{D}$ of $\mathrm{X}$, denoted $D=A \cap B$ identified by the minimum ( $\min$ ) value between $A(x)$ and $B(x)$, such that for each $\mathrm{x}$ in $\mathrm{X}$ :

$$
D(\mathrm{x})=\min [A(x), B(x)]
$$

Fuzzy relations are another important concept in fuzzy sets. A fuzzy relation $\mathrm{R}$ between two crisp sets $X=\{x\}$ and $Y=\{y\}$ is defined as a fuzzy set in the Cartesian product $X \times Y$, i.e.,

$$
\mathrm{R}=\left\{\mu_{\mathrm{R}}(\mathrm{x}, \mathrm{y}) /(\mathrm{x}, \mathrm{y})\right\}
$$

for each $(\mathrm{x}, \mathrm{y}) \in \mathrm{X} \times \mathrm{Y}$, where $\mu_{\mathrm{R}}(\mathrm{x}, \mathrm{y}): \mathrm{X} \times \mathrm{Y} \rightarrow[0,1]$ is the membership function of the fuzzy relation $\mathrm{R}$, and $\mu_{\mathrm{R}}(\mathrm{x}, \mathrm{y}) \in[0,1]$ gives the degree to which the elements $x \in X$ and $y \in Y$ are in relation $\mathrm{R}$ to each other. Since this basic type of a fuzzy relation is defined in the Cartesian product of two sets, such a fuzzy relation is sometimes called a binary fuzzy relation. However, this concept could be generalized to $\mathrm{n}$ dimensions of fuzzy relations. A fuzzy relation describe a partial relationship between elements of some sets, as opposed to a precise one in the case of a crisp relation in which any elements can either be related or not. The gradual relationships, from 1 for being fully in relation to 0 for not being in relation at all, are concerned in fuzzy relation. Fuzzy relations are represented in a matrix form, which usually simplifies the composition of fuzzy relation methods. 
The max-min composition is a useful composition of fuzzy relations. The max-min composition of two fuzzy relations $\mathrm{R}$ in $X \times Y$ and $\mathrm{S}$ in $Y \times Z$, written $R o_{\max -\min } S$ is defined as a fuzzy relation in $X \times Z$ such that

$$
\mu_{\mathrm{Ro}_{\max -\min } \mathrm{S}}(\mathrm{x}, \mathrm{y})=\max \left[\min \left(\mu_{\mathrm{R}}(\mathrm{x}, \mathrm{y}), \mu_{\mathrm{S}}(\mathrm{y}, \mathrm{z})\right)\right]
$$

for each $x \in \mathrm{X}$ and $z \in Z$. The mathematical operation expressed (5) is similar to a multiplication of matrices, where each matrix represents a fuzzy relation [11-13].

\subsection{Fuzzy Expert System}

A fuzzy expert system is an expert system that profits fuzzy logic instead of Boolean logic. Also, a collection of membership functions and rules is called a fuzzy expert. The conventional expert systems are symbolic reasoning engines but fuzzy expert systems usually use the numerical processing.

The rules in a fuzzy expert system are usually of a form similar to the following:

$$
\text { if } x \text { is low and } y \text { is high then } z=\text { medium }
$$

where $\mathrm{x}$ and $\mathrm{y}$ are input variables and known data values. $\mathrm{z}$ is an output variable which must be computed. "Low", "high" and "medium" are the membership functions defined on $\mathrm{x}, \mathrm{y}$, and $\mathrm{z}$, respectively. The part of the rule between the "if " and "then " is the rule's "premise" or "antecedent". This is a fuzzy logic expression that describes to what degree the rule is applicable. The part of the rule following the "then" is the rule's "conclusion" or "consequent". This part of the rule assigns a membership function to each of one or more output variables. The tools for working with fuzzy expert systems often allow more than one conclusion per rule.

The fuzzy expert systems often have more than one rule. The entire collection of rules is called a "rule base" or "knowledge base" [14].

\section{METHODS}

Homeopaths often use two methods for selection of remedy: "Summation" and "Elimination". In summation method, homeopath assigns intensity to each selected rubric and degree of remedies of the rubric is multiplied by the determined intensity. Ultimately, the product results are added to create index for each remedy. For determining a remedy, the homeopath searches in remedies with greater index to find the best simillimum. The problem of this method is that proving of various remedies are not complete and the same degree of remedies does not have similar meaning.

In elimination method, a rubric which is big \& solid, and everyone has to agree on is selected i.e. it should be an objective rubric and not subjective. The selected rubrics are eliminated after their selection. The problem of this method is that if a small rubric is selected and eliminated in first steps, then patient's remedy may be lost.

Selection of remedy for a patient is the most important activity that is done by homeopath. After a patient refers to homeopath and homeopath does case taking, (s)he selects the best rubrics for the patient. Uncertain selection of rubrics sometimes happens due to the mistaken decision about the remedy of the patient. In this paper, we study machine learning methods to help the homeopath to decrease errors in homeopathy.

\subsection{Decision tree}

In decision tree method, several patients with their rubrics and results should be selected and used for creation of decision tree. Trees are created once and then use for diagnosis.

In homeopathy, each patient is an individual and two patients with the same problem may have two different remedies. As a result, we cannot select cases for creation of decision tree. Therefore, decision tree method cannot be used for selection of remedy in homeopathy.

\subsection{Fuzzy Expert System}

Fuzzy expert system is an expert system that uses fuzzy content. For this paper, we define four states for input and four states for knowledge base. Output of the system is remedies purposed to homeopath for the patient.

In proposed system, we consider four states for rubrics in repertory: never, sometimes, often, and always. Membership functions of these states are shown in Fig1.
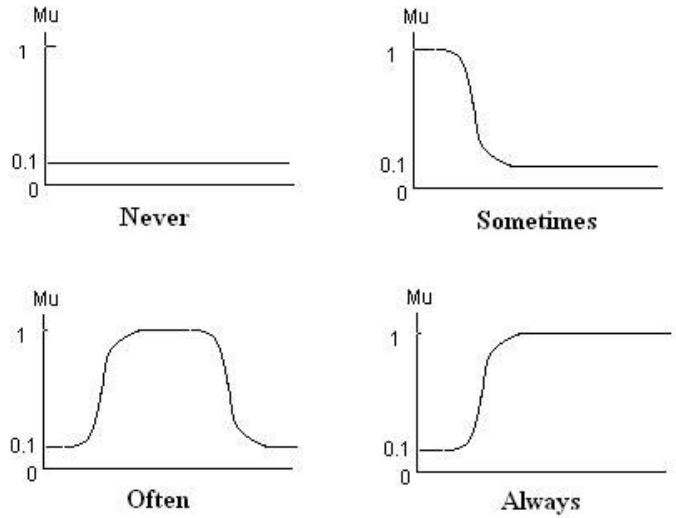

Fig 1: States for rubrics in repertory (Knowledge base)

In Fig 1, "never" is a fixed value slightly higher than zero because remedy may have one rubric but yet, it shows the rubric in its proving is not complete. "Sometimes" is more possible of accession in less numbers. "Often" and "always" are more possible of accession in normal and more numbers.

Also we consider four states for selected rubrics for a patient: none, low, medium, and high. Fig 2 show states for the selected rubrics for a patient.
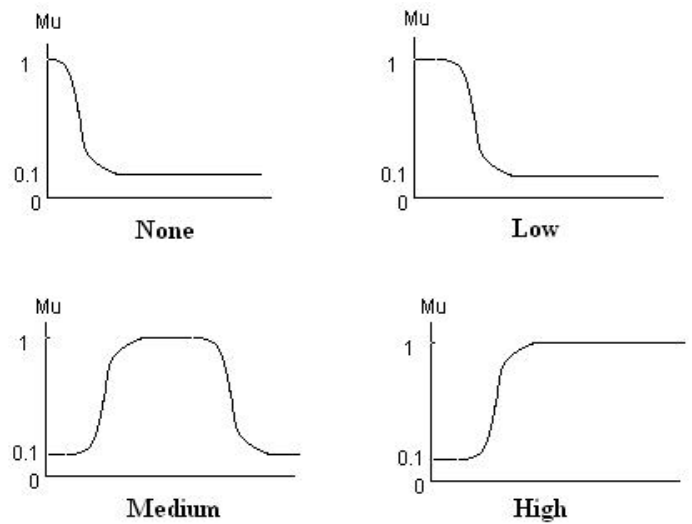

Fig 2: States for selected rubrics for a patient (Input) 
In Fig 2, "none" has less probability because a patient may not have a symptom but it is accessed if required conditions are provided. "Low" has low intensity or rareness of symptom. "Medium" and "High" are intensity or times of accession.

To find the result, output of the first phase is calculated with use of rules and their membership functions. For each selected rubrics for the patient, homeopath considers one of the states of Fig 2 that is compatible with state of the input. Curve of the selected rubric is adapted on the curve of the intensity of rubric in repertory for all remedies. For each remedy, max point of cross of curves is selected. This phase is done for all selected rubrics.

In the next phase, for each remedy, the product of acquired values of maximums in the first phase is calculated. The score of each remedy is the value of acquired product of the last phase. This style for selection is called "max-prod reasoning". Scores are multiplied by

$100 \times \exp (($ No. of selected rubrics $/ 10)-1)$ to set the $\log$ of results between -1 and 1 .

Finally, a threshold is used and the remedies with values less than this threshold are deleted and other remedies remain This set of remedies of remainder is final result of this system.

Every patient may be have capacity of a rubric but yet don't access. As a result, membership function isn't equal by zero, this case is considered. This subject is true about rubrics which are not yet observing for a remedy and cause of that is that proving are not complete. These are benefits of this method.

\section{DISCUSSION}

The Vithoulkas Expert System imitates the line of thought of Prof. Vithoulkas and therefore it is not useful for the homeopaths that don't work with Vithoulkas's opinion. As a result, some of the homeopaths work with summation or elimination methods.

The problem of summation method is that proving of various remedies are not complete and same degree of remedies does not have similar meaning. The problem with elimination method is that if a small rubric is selected and eliminated in first steps, the patient's remedy might be lost.

The proposed fuzzy expert system has benefits. As a remedy may have one rubric but yet, the rubric shown in its proving is not complete, we consider a fixed value which is not equal to zero for that. We also consider a membership function for not existing symptoms because it happens that a patient does not have a symptom but it is accessed if the required conditions are provided.

Proposed fuzzy expert system method helps to prevent elimination of remedies unintentionally. In elimination method, the sequence of elimination is very important and if one rubric is eliminated earlier, it may be lost from the patent's remedy.

Every patient may have capacity of a rubric but yet don't access it or the rubrics may not be observed for a remedy because its proving aren't complete yet. For the mention subjects, we consider membership function that is not equal by zero. This has benefit for the purposed method.

With smaller thresholds, more remedies are shown and less selected remedies are obtained with large threshold.

\section{CONCLUSION}

Nowadays, artificial intelligence methods are widely used to help experts. In this work we investigated application of fuzzy expert system and decision trees for homeopathy. The decision tree showed poor performance while we could get useful results from a fuzzy expert system.

Homeopathy is very sensitive to terms that a patient expresses therefore a fuzzy expert system can help the homeopath to select the proper remedy. Although, the implementation of this system requires takes long time, this method is very useful in practice.

To continue this work, it is proposed to investigate rubrics and select the most useful ones. Then, a fuzzy expert system can be trained using selected rubrics to reduce the implementation time.

\section{REFERENCES}

[1] I. V. Polony, "The use of manual and computer aided search methods in the homeopathic repertory," AEON GROUP, pp. 1-6, 2005.

[2] "Readings in machine learning," J. W. Shavlik and T. G. Dietterich, Eds., ed: Morgan Kaufmann Publishers, San Mateo, California, 1990.

[3] I. Kononenko, "Machine learning for medical diagnosis: history, state of the art and perspective," Artificial Intelligence in Medicine, vol. 23, pp. 89-109, 2001.

[4] I. Kononenko, et al., "Application of machine learning to medical diagnosis," in Machine Learning and Data Mining: Methods and Applications, R. S. Michalski, et al., Eds., ed: John Wiley \& Sons Ltd, 1997.

[5] M. A. M. Reis, et al., "Fuzzy expert system in the prediction of neonatal resuscitation," Brazilian Journal of Medical and Biological Research, vol. 37, pp. 755764, 2004.

[6] H. Teodorescu, et al., "Fuzzy and Neuro-Fuzzy Systems in Medicine," CRC Press, Boca Raton, FL, USA, 1999.

[7] K. P. Adlassnig, "A fuzzy logical model of computerassisted medical diagnosis," Methods Inf Med, vol. 19, pp. 141-148, 1980.

[8] G. Vithoulkas. The Vithoulkas Expert System (V.E.S.).

[9] (2011). Decision tree learning. Available: http://en.wikipedia.org/wiki/

[10] L. A. Zadeh, "Fuzzy sets," Information and Control, vol. 8, pp. 338-353, 1965.

[11] D. M. Velasevic, et al., "A fuzzy sets theory application in determining the severity of respiratory failure," International Journal of Medical Informatics, vol. 63, pp. 101-107, 2001.

[12] G. J. Klir and T. A. Folger, Fuzzy Sets, Uncertainty, and Information. Englewood Cliffs, NJ: Prentice Hall, 1988.

[13] W. Pedrycz and F. Gomide, An Introduction to Fuzzy Sets: Analysis and Design. Massachusetts, London: A Bradford Book, The MIT Press Cambridge, 1998.

[14] (2011). Fuzzy Expert Systems. Available: http://www.austinlinks.com/Fuzzy/expert-systems.html 